\title{
Differential sandwich results for Wanas operator of analytic functions
}

\author{
ABBAS KAREEM WANAS, \\ Gangadharan Murugusundaramoorthy
}

\begin{abstract}
In the present article, we determine some subordination and superordination results involving Wanas operator for certain normalized analytic functions defined in the unit disk $\mathbb{U}$. These results are applied to establish sandwich results. Our results extend corresponding previously known results.
\end{abstract}

\section{INTRODUCTION}

Denote by $H=H(\mathbb{U})$ the collection of analytic functions in the unit disk $\mathbb{U}=\{z \in \mathbb{C}:|z|<1\}$ and assume that $H[a, n]$ be the subclass of $H$ consisting of functions of the form:

$$
f(z)=a+a_{n} z^{n}+a_{n+1} z^{n+1}+\ldots \quad(a \in \mathbb{C}, n \in \mathbb{N}=\{1,2, \ldots\}) .
$$

Also, let $\mathcal{A}$ be the subclass of $H$ consisting of functions of the form:

$$
f(z)=z+\sum_{n=2}^{\infty} a_{n} z^{n} .
$$

Now we recall the principal of subordination between analytic functions, let the functions $f$ and $g$ be analytic in $\mathbb{U}$, we say that the function $f$ is subordinate to $g$, if there exists a Schwarz function $w$ analytic in $\mathbb{U}$ with $w(0)=0$ and $|w(z)|<1(z \in \mathbb{U})$ such that $f(z)=g(w(z))$. This subordination is indicated by $f \prec g$ or $f(z) \prec g(z)(z \in \mathbb{U})$. Furthermore, if the function $g$ is univalent in $\mathbb{U}$, then we have the following equivalent (see [8]), $f(z) \prec g(z) \Longleftrightarrow f(0)=g(0)$ and $f(\mathbb{U}) \subset g(\mathbb{U})$.

Let $\xi, h \in H$ and $\psi(r, s, t ; z): \mathbb{C}^{3} \times \mathbb{U} \rightarrow \mathbb{C}$. If $\xi$ and

$$
\psi\left(\xi(z), z \xi^{\prime}(z), z^{2} \xi^{\prime \prime}(z) ; z\right)
$$

2010 Mathematics Subject Classification. Primary: 30C45; Secondary: 30C80.

Key words and phrases. Analytic function, differential subordination, differential superoordination, dominant, subordinant, Wanas operator.

Full paper. Received 4 November 2019, revised 21 January 2020, accepted 23 January 2020, available online 29 February 2020. 
are univalent functions in $\mathbb{U}$ and if $\xi$ satisfies the second-order differential superordination

$$
h(z) \prec \psi\left(\xi(z), z \xi^{\prime}(z), z^{2} \xi^{\prime \prime}(z) ; z\right),
$$

then $\xi$ is called a solution of the differential superordination (2). (If $f$ is subordinate to $g$, then $g$ is superordinate to $f$ ). An analytic function $q$ is called a subordinat of (2), if $q \prec \xi$ for all $\xi$ satisfying (2). An univalent subordinant $\widetilde{q}$ that satisfies $q \prec \widetilde{q}$ for all the subordinates $q$ of $(2)$ is called the best subordinant.

For $\alpha \in \mathbb{R}, \beta \geq 0$ with $\alpha+\beta>0, m, \delta \in \mathbb{N}_{0}=\mathbb{N} \cup\{0\}$ and $f \in \mathcal{A}$, the Wanas operator $W_{\alpha, \beta}^{k, \delta}: \mathcal{A} \rightarrow \mathcal{A}$ (see [24]) is defined by

$$
W_{\alpha, \beta}^{k, \delta} f(z)=z+\sum_{n=2}^{\infty}\left[\sum_{m=1}^{k}\left(\begin{array}{c}
k \\
m
\end{array}\right)(-1)^{m+1}\left(\frac{\alpha^{m}+n \beta^{m}}{\alpha^{m}+\beta^{m}}\right)\right]^{\delta} a_{n} z^{n} .
$$

Remark 1. It should be remarked that the operator $W_{\alpha, \beta}^{k, \delta}$ generalizes some known operators considered earlier:

(1) For $k=1$, the operator $W_{\alpha, \beta}^{1, \delta} \equiv I_{\alpha, \beta}^{\delta}$ was introduced and studied by Swamy [22],

(2) For $k=\beta=1, \delta=-\mu, \operatorname{Re}(\mu)>1$ and $\alpha \in \mathbb{C} \backslash \mathbb{Z}_{0}^{-}$, the operator $W_{\alpha, 1}^{1,-\mu} \equiv J_{\mu, \alpha}$ was investigated by Srivastava and Attiya [16]. The operator $J_{\mu, \alpha}$ is now popularly known in the literature as the Srivastava-Attiya operator. Various applications of the SrivastavaAttiya operator are found in $[15,17,18,19,20]$ and in the references cited in each of these earlier works,

(3) For $k=\beta=1$ and $\alpha>-1$, the operator $W_{\alpha, 1}^{1, \delta} \equiv I_{\alpha}^{\delta}$ was investigated by Cho and Srivastava [6],

(4) For $k=\alpha=\beta=1$, the operator $W_{1,1}^{1, \delta} \equiv I^{\delta}$ was considered by Uralegaddi and Somanatha [23],

(5) For $k=\alpha=\beta=1, \delta=-\sigma$ and $\sigma>0$, the operator $W_{1,1}^{1,-\sigma} \equiv I^{\sigma}$ was introduced by Jung et al. [7]. The operator $I^{\sigma}$ is the Jung-KimSrivastava integral operator,

(6) For $k=\beta=1, \delta=-1$ and $\alpha>-1$, the operator $W_{\alpha, 1}^{1,-1} \equiv L_{\alpha}$ was studied by Bernardi [4],

(7) For $\alpha=0, k=\beta=1$ and $\delta=-1$, the operator $W_{0,1}^{1,-1} \equiv u$ was investigated by Alexander [1],

(8) For $k=1, \alpha=1-\beta$ and $\beta \geq 0$, the operator $W_{1-\beta, \beta}^{1, \delta} \equiv D_{\beta}^{\delta}$ was given by Al-Oboudi [2],

(9) For $k=1, \alpha=0$ and $\beta=1$, the operator $W_{0,1}^{1, \delta} \equiv S^{\delta}$ was considered by Sălăgean [13]. 
It is readily verified from $(3)$ that

$$
\begin{aligned}
z\left(W_{\alpha, \beta}^{k, \delta} f(z)\right)^{\prime} & =\left[\sum_{m=1}^{k}\left(\begin{array}{c}
k \\
m
\end{array}\right)(-1)^{m+1}\left(\left(\frac{\alpha}{\beta}\right)^{m}+1\right)\right] W_{\alpha, \beta}^{k, \delta+1} f(z) \\
& -\left[\sum_{m=1}^{k}\left(\begin{array}{c}
k \\
m
\end{array}\right)(-1)^{m+1}\left(\frac{\alpha}{\beta}\right)^{m}\right] W_{\alpha, \beta}^{k, \delta} f(z) .
\end{aligned}
$$

Very recently, Rahrovi [12], Attiya and Yassen [3], Seoudy [14], Wanas and Majeed [25] and Srivastava and Wanas [21] have obtained sandwich results for certain classes of analytic functions. Motivated by aforementioned works to investigate sufficient condition for $f$ based on Wanas differential operator we define a new subclasses of normalized analytic functions satisfying the following:

$$
q_{1}(z) \prec\left(\frac{W_{\alpha, \beta}^{k, \delta} f(z)}{z}\right)^{\gamma} \prec q_{2}(z)
$$

and

$$
q_{1}(z) \prec\left(\frac{W_{\alpha, \beta}^{k, \delta+1} f(z)}{W_{\alpha, \beta}^{k, \delta} f(z)}\right)^{\gamma} \prec q_{2}(z),
$$

where $q_{1}$ and $q_{2}$ are given univalent functions in $\mathbb{U}$ with $q_{1}(0)=q_{2}(0)=1$. To establish our main results, we need the following definition and lemmas.

Definition $1([8])$. Denote by $Q$ the set of all functions $f$ that are analytic and injective on $\overline{\mathbb{U}} \backslash E(f)$, where

$$
E(f)=\left\{\zeta \in \partial \mathbb{U}: \lim _{z \rightarrow \zeta} f(z)=\infty\right\}
$$

and are such that $f^{\prime}(\zeta) \neq 0$ for $\zeta \in \partial \mathbb{U} \backslash E(f)$.

Lemma 1 ([8]). Let $q$ be univalent in the unit disk $\mathbb{U}$ and let $\theta$ and $\phi$ be analytic in a domain $D$ containing $q(\mathbb{U})$ with $\phi(w) \neq 0$ when $w \in q(\mathbb{U})$. Set $Q(z)=z q^{\prime}(z) \phi(q(z))$ and $h(z)=\theta(q(z))+Q(z)$. Suppose that

(1) $Q(z)$ is starlike univalent in $\mathbb{U}$,

(2) $\Re\left(\frac{z h^{\prime}(z)}{Q(z)}\right)>0$ for $z \in \mathbb{U}$.

If $\xi$ is analytic in $\mathbb{U}$, with $\xi(0)=q(0), \xi(\mathbb{U}) \subset D$ and

$$
\theta(\xi(z))+z \xi^{\prime}(z) \phi(\xi(z)) \prec \theta(q(z))+z q^{\prime}(z) \phi(q(z)),
$$

then $\xi \prec q$ and $q$ is the best dominant of (5).

Lemma $2([9])$. Let $q$ be a convex univalent function in $\mathbb{U}$ and let $\mu \in \mathbb{C}$, $\nu \in \mathbb{C} \backslash\{0\}$ with

$$
\Re\left(1+\frac{z q^{\prime \prime}(z)}{q^{\prime}(z)}\right)>\max \left\{0,-R e\left(\frac{\mu}{\nu}\right)\right\} .
$$


If $\xi$ is analytic in $\mathbb{U}$ and

$$
\mu \xi(z)+\nu z \xi^{\prime}(z) \prec \mu q(z)+\nu z q^{\prime}(z),
$$

then $\xi \prec q$ and $q$ is the best dominant of (6).

Lemma $3([9])$. Let $q$ be convex univalent in $\mathbb{U}$ and let $\nu \in \mathbb{C}$. Further assume that $\Re(\nu)>0$. If $\xi \in H[q(0), 1] \cap Q$ and $\xi(z)+\nu z \xi^{\prime}(z)$ is univalent in $\mathbb{U}$, then

$$
q(z)+\nu z q^{\prime}(z) \prec \xi(z)+\nu z \xi^{\prime}(z),
$$

which implies that $q \prec \xi$ and $q$ is the best subordinant of (7).

Lemma 4 ([5]). Let $q$ be convex univalent in the unit disk $\mathbb{U}$ and let $\theta$ and $\phi$ be analytic in a domain D containing $q(\mathbb{U})$. Suppose that

(1) $\Re\left(\frac{\theta^{\prime}(q(z))}{\phi(q(z))}\right)>0$ for $z \in \mathbb{U}$,

(2) $Q(z)=z q^{\prime}(z \phi(q(z)))$ is starlike univalent in $\mathbb{U}$.

If $\xi \in H[q(0), 1] \cap Q$, with $\xi(\mathbb{U}) \subset D, \phi(\xi(z))+z \xi^{\prime}(z) \phi(\xi(z))$ is univalent in $\mathbb{U}$ and

$$
\theta(q(z))+z q^{\prime}(z) \phi(q(z)) \prec \theta(\xi(z))+z \xi^{\prime}(z) \phi(\xi(z)),
$$

then $q \prec \xi$ and $q$ is the best subordinant of (8).

\section{Main Results}

Theorem 1. Let $q$ be convex univalent in $\mathbb{U}$ with $q(0)=1, \sigma \in \mathbb{C} \backslash\{0\}$, $\gamma>0$ and suppose that $q$ satisfies

$$
\Re\left(1+\frac{z q^{\prime \prime}(z)}{q^{\prime}(z)}\right)>\max \left\{0,-\Re\left(\frac{\gamma}{\sigma}\right)\right\} .
$$

If $f \in \mathcal{A}$ satisfies the subordination

$$
\begin{aligned}
& {\left[1-\sum_{m=1}^{k}\left(\begin{array}{c}
k \\
m
\end{array}\right)(-1)^{m+1}\left(\left(\frac{\alpha}{\beta}\right)^{m}+1\right)\right]\left(\frac{W_{\alpha, \beta}^{k, \delta} f(z)}{z}\right)^{\gamma}} \\
& +\sum_{m=1}^{k}\left(\begin{array}{c}
k \\
m
\end{array}\right)(-1)^{m+1}\left(\left(\frac{\alpha}{\beta}\right)^{m}+1\right)\left(\frac{W_{\alpha, \beta}^{k, \delta} f(z)}{z}\right)^{\gamma}\left(\frac{W_{\alpha, \beta}^{k, \delta+1} f(z)}{W_{\alpha, \beta}^{k, \delta} f(z)}\right) \\
& \prec q(z)+\frac{\sigma}{\gamma} z q^{\prime}(z),
\end{aligned}
$$

then

$$
\left(\frac{W_{\alpha, \beta}^{k, \delta} f(z)}{z}\right)^{\gamma} \prec q(z)
$$

and $q$ is the best dominant of (10). 
Proof. Define the function $\xi$ by

$$
\xi(z)=\left(\frac{W_{\alpha, \beta}^{k, \delta} f(z)}{z}\right)^{\gamma}, \quad(z \in \mathbb{U}) .
$$

Differentiating (12) logarithmically with respect to $z$, we get

$$
\frac{z \xi^{\prime}(z)}{\xi(z)}=\gamma\left(\frac{z\left(W_{\alpha, \beta}^{k, \delta} f(z)\right)^{\prime}}{W_{\alpha, \beta}^{k, \delta} f(z)}-1\right)
$$

Now, in view of (4), we obtain the following subordination

$$
\frac{z \xi^{\prime}(z)}{\xi(z)}=\sum_{m=1}^{k}\left(\begin{array}{c}
k \\
m
\end{array}\right)(-1)^{m+1}\left(\left(\frac{\alpha}{\beta}\right)^{m}+1\right)\left(\frac{W_{\alpha, \beta}^{k, \delta+1} f(z)}{W_{\alpha, \beta}^{k, \delta} f(z)}-1\right) .
$$

Therefore,

$$
\begin{aligned}
\frac{z \xi^{\prime}(z)}{\gamma} & =\sum_{m=1}^{k}\left(\begin{array}{c}
k \\
m
\end{array}\right)(-1)^{m+1}\left(\left(\frac{\alpha}{\beta}\right)^{m}+1\right) \times \\
& \times\left(\frac{W_{\alpha, \beta}^{k, \delta} f(z)}{z}\right)^{\gamma}\left(\frac{W_{\alpha, \beta}^{k, \delta+1} f(z)}{W_{\alpha, \beta}^{k, \delta} f(z)}-1\right) .
\end{aligned}
$$

The subordination (10) from the hypothesis becomes

$$
\xi(z)+\frac{\sigma}{\gamma} z \xi^{\prime}(z) \prec q(z)+\frac{\sigma}{\gamma} z q^{\prime}(z) .
$$

Hence, an application of Lemma 2 with $\mu=1$ and $\nu=\frac{\sigma}{\gamma}$, we obtain (11).

Theorem 2. Let $\eta, \tau \in \mathbb{C}, \gamma>0, \lambda \in \mathbb{C} \backslash\{0\}$ and $q$ be convex univalent in $\mathbb{U}$ with $q(0)=1, q(z) \neq 0(z \in \mathbb{U})$ and assume that $q$ satisfies

$$
\Re\left(1+\frac{\tau}{\lambda} q(z)+\frac{z q^{\prime \prime}(z)}{q^{\prime}(z)}-\frac{z q^{\prime}(z)}{q(z)}\right)>0 .
$$

Suppose that $\frac{z q^{\prime}(z)}{q(z)}$ is starlike univalent in $\mathbb{U}$. If $f \in \mathcal{A}$ satisfies

$$
\Omega(\eta, \tau, \gamma, \lambda, k, \delta, \alpha, \beta ; z) \prec \eta+\tau q(z)+\lambda \frac{z q^{\prime}(z)}{q(z)},
$$

where

$$
\begin{aligned}
& \Omega(\eta, \tau, \gamma, \lambda, k, \delta, \alpha, \beta ; z)=\eta+\tau\left(\frac{W_{\alpha, \beta}^{k, \delta+1} f(z)}{W_{\alpha, \beta}^{k, \delta} f(z)}\right)^{\gamma} \\
& +\gamma \lambda \sum_{m=1}^{k}\left(\begin{array}{c}
k \\
m
\end{array}\right)(-1)^{m+1}\left(\left(\frac{\alpha}{\beta}\right)^{m}+1\right)\left(\frac{W_{\alpha, \beta}^{k, \delta+2} f(z)}{W_{\alpha, \beta}^{k, \delta+1} f(z)}-\frac{W_{\alpha, \beta}^{k, \delta+1} f(z)}{W_{\alpha, \beta}^{k, \delta} f(z)}\right),
\end{aligned}
$$


then

$$
\left(\frac{W_{\alpha, \beta}^{k, \delta+1} f(z)}{W_{\alpha, \beta}^{k, \delta} f(z)}\right)^{\gamma} \prec q(z)
$$

and $q$ is the best dominant of (14).

Proof. Define the function $\xi$ by

$$
\xi(z)=\left(\frac{W_{\alpha, \beta}^{k, \delta+1} f(z)}{W_{\alpha, \beta}^{k, \delta} f(z)}\right)^{\gamma}, \quad(z \in \mathbb{U}) .
$$

By a straightforward computation and using (4), we have

$$
\eta+\tau \xi(z)+\lambda \frac{z \xi^{\prime}(z)}{\xi(z)}=\Omega(\eta, \tau, \gamma, \lambda, k, \delta, \alpha, \beta ; z)
$$

where $\Omega(\eta, \tau, \gamma, \lambda, k, \delta, \alpha, \beta ; z)$ is given by (15). From (14) and (17), we obtain

By setting

$$
\eta+\tau \xi(z)+\lambda \frac{z \xi^{\prime}(z)}{\xi(z)} \prec \eta+\tau q(z)+\lambda \frac{z q^{\prime}(z)}{q(z)} .
$$

$$
\theta(w)=\eta+\tau w \text { and } \phi(w)=\frac{\lambda}{w}, w \neq 0,
$$

we see that $\theta(w)$ is analytic in $\mathbb{C}, \phi(w)$ is analytic in $\mathbb{C} \backslash\{0\}$ and that $\phi(w) \neq 0, w \in \mathbb{C} \backslash\{0\}$. Also, we get

$$
Q(z)=z q^{\prime}(z) \phi(q(z))=\lambda \frac{z q^{\prime}(z)}{q(z)}
$$

and

$$
h(z)=\theta(q(z))+Q(z)=\eta+\tau q(z)+\lambda \frac{z q^{\prime}(z)}{q(z)} .
$$

It is clear that $Q(z)$ is starlike univalent in $\mathbb{U}$,

$$
\Re\left(\frac{z h^{\prime}(z)}{Q(z)}\right)=\Re\left(1+\frac{\tau}{\lambda} q(z)+\frac{z q^{\prime \prime}(z)}{q^{\prime}(z)}-\frac{z q^{\prime}(z)}{q(z)}\right)>0 .
$$

Thus, by Lemma 1 , we get $\xi(z) \prec q(z)$. By using (16), we obtain the desired result.

Theorem 3. Let $q$ be convex univalent in $\mathbb{U}$ with $q(0)=1, \gamma>0$ and $\Re(\sigma)>0$. Let $f \in \mathcal{A}$ satisfies

$$
\left(\frac{W_{\alpha, \beta}^{k, \delta} f(z)}{z}\right)^{\gamma} \in H[q(0), 1] \cap Q
$$

and

$$
\left[1-\sigma \sum_{m=1}^{k}\left(\begin{array}{c}
k \\
m
\end{array}\right)(-1)^{m+1}\left(\left(\frac{\alpha}{\beta}\right)^{m}+1\right)\right]\left(\frac{W_{\alpha, \beta}^{k, \delta} f(z)}{z}\right)^{\gamma}
$$




$$
+\sigma \sum_{m=1}^{k}\left(\begin{array}{c}
k \\
m
\end{array}\right)(-1)^{m+1}\left(\left(\frac{\alpha}{\beta}\right)^{m}+1\right)\left(\frac{W_{\alpha, \beta}^{k, \delta} f(z)}{z}\right)^{\gamma}\left(\frac{W_{\alpha, \beta}^{k, \delta+1} f(z)}{W_{\alpha, \beta}^{k, \delta} f(z)}\right)
$$

be univalent in $\mathbb{U}$. If

$$
\begin{aligned}
& q(z)+\frac{\sigma}{\gamma} z q^{\prime}(z) \\
& \prec\left[1-\sigma \sum_{m=1}^{k}\left(\begin{array}{c}
k \\
m
\end{array}\right)(-1)^{m+1}\left(\left(\frac{\alpha}{\beta}\right)^{m}+1\right)\right]\left(\frac{W_{\alpha, \beta}^{k, \delta} f(z)}{z}\right)^{\gamma} \\
& +\sigma \sum_{m=1}^{k}\left(\begin{array}{c}
k \\
m
\end{array}\right)(-1)^{m+1}\left(\left(\frac{\alpha}{\beta}\right)^{m}+1\right)\left(\frac{W_{\alpha, \beta}^{k, \delta} f(z)}{z}\right)^{\gamma} \times \\
& \times\left(\frac{W_{\alpha, \beta}^{k, \delta+1} f(z)}{W_{\alpha, \beta}^{k, \delta} f(z)}\right),
\end{aligned}
$$

then

$$
q(z) \prec\left(\frac{W_{\alpha, \beta}^{k, \delta} f(z)}{z}\right)^{\gamma}
$$

and $q$ is the best subordinant of (18).

Proof. Let $\xi$ be defined by (12), then differentiating $\xi$ with respect to $z$, we get

$$
\frac{z \xi^{\prime}(z)}{\xi(z)}=\gamma\left(\frac{z\left(W_{\alpha, \beta}^{k, \delta} f(z)\right)^{\prime}}{W_{\alpha, \beta}^{k, \delta} f(z)}-1\right)
$$

By using (4) for $\left(W_{\alpha, \beta}^{k, \delta} f(z)\right)^{\prime}$, in (20), we have

$$
\begin{aligned}
& {\left[1-\sigma \sum_{m=1}^{k}\left(\begin{array}{c}
k \\
m
\end{array}\right)(-1)^{m+1}\left(\left(\frac{\alpha}{\beta}\right)^{m}+1\right)\right]\left(\frac{W_{\alpha, \beta}^{k, \delta} f(z)}{z}\right)^{\gamma}} \\
& +\sigma \sum_{m=1}^{k}\left(\begin{array}{c}
k \\
m
\end{array}\right)(-1)^{m+1}\left(\left(\frac{\alpha}{\beta}\right)^{m}+1\right)\left(\frac{W_{\alpha, \beta}^{k, \delta} f(z)}{z}\right)^{\gamma} \times \\
& \times\left(\frac{W_{\alpha, \beta}^{k, \delta+1} f(z)}{W_{\alpha, \beta}^{k, \delta} f(z)}\right)=\xi(z)+\frac{\sigma}{p \gamma} z \xi^{\prime}(z) .
\end{aligned}
$$

From (18) and (21), we get

$$
q(z)+\frac{\sigma}{\gamma} z q^{\prime}(z) \prec \xi(z)+\frac{\sigma}{\gamma} z \xi^{\prime}(z) .
$$

Hence, by using Lemma 3 with $\mu=1$ and $\nu=\frac{\sigma}{\gamma}$, we obtain (19). 
Theorem 4. Let $\eta \in \mathbb{C}, \gamma>0, \lambda \in \mathbb{C} \backslash\{0\}$ and $q$ be convex univalent in $\mathbb{U}$ with $q(0)=1, q(z) \neq 0(z \in \mathbb{U})$ and assume that $q$ satisfies

$$
\Re\left(\frac{\tau}{\lambda} q(z)\right)>0 .
$$

Suppose that $\frac{z q^{\prime}(z)}{q(z)}$ is starlike univalent in $\mathbb{U}$. If $f \in \mathcal{A}$ satisfies

$$
\left(\frac{W_{\alpha, \beta}^{k, \delta+1} f(z)}{W_{\alpha, \beta}^{k, \delta} f(z)}\right)^{\gamma} \in H[q(0), 1] \cap Q
$$

and $\Omega(\eta, \tau, \gamma, \lambda, k, \delta, \alpha, \beta ; z)$ is univalent in $\mathbb{U}$, where $\Omega(\eta, \tau, \gamma, \lambda, k, \delta, \alpha, \beta ; z)$ is given by (15). If

$$
\eta+\tau q(z)+\lambda \frac{z q^{\prime}(z)}{q(z)} \prec \Omega(\eta, \tau, \gamma, \lambda, k, \delta, \alpha, \beta ; z),
$$

then

$$
q(z) \prec\left(\frac{W_{\alpha, \beta}^{k, \delta+1} f(z)}{W_{\alpha, \beta}^{k, \delta} f(z)}\right)^{\gamma}
$$

and $q$ is the best subordinant of (23).

Proof. Assume that the function $\xi$ be defined by (16). By a straightforward computation, we have

$$
\Omega(\eta, \tau, \gamma, \lambda, k, \delta, \alpha, \beta ; z)=\eta+\tau \xi(z)+\lambda \frac{z \xi^{\prime}(z)}{\xi(z)},
$$

where $\Omega(\eta, \tau, \gamma, \lambda, k, \delta, \alpha, \beta ; z)$ is given by (15). From (23) and (24), we obtain

$$
\eta+\tau q(z)+\lambda \frac{z q^{\prime}(z)}{q(z)} \prec \eta+\tau \xi(z)+\lambda \frac{z \xi^{\prime}(z)}{\xi(z)} .
$$

By setting $\theta(w)=\eta+\tau w$ and $\phi(w)=\frac{\lambda}{w}, w \neq 0$, we see that $\theta(w)$ is analytic in $\mathbb{C}, \phi(w)$ is analytic in $\mathbb{C} \backslash\{0\}$ and that $\phi(w) \neq 0, w \in \mathbb{C} \backslash\{0\}$. Also, we get

$$
Q(z)=z q^{\prime}(z) \phi(q(z))=\lambda \frac{z q^{\prime}(z)}{q(z)} .
$$

It is clear that $Q(z)$ is starlike univalent in $\mathbb{U}$,

$$
\Re\left(\frac{\theta^{\prime}(q(z))}{\phi(q(z))}\right)=\Re\left(\frac{\tau}{\lambda} q(z)\right)>0 .
$$

Thus, by Lemma 4 , we get $q(z) \prec \xi(z)$. By using (16), we obtain the desired result.

Concluding the results of differential subordination and superordination, we state the following "sandwich results". 
Theorem 5. Let $q_{1}$ and $q_{2}$ be convex univalent in $\mathbb{U}$ with $q_{1}(0)=q_{2}(0)=1$. Suppose $q_{2}$ satisfies (9), $\gamma>0$ and $\Re(\sigma)>0$. Let $f \in \mathcal{A}$ satisfies

$$
\left(\frac{W_{\alpha, \beta}^{k, \delta} f(z)}{z}\right)^{\gamma} \in H[1,1] \cap Q
$$

and

$$
\begin{aligned}
& {\left[1-\sigma \sum_{m=1}^{k}\left(\begin{array}{c}
k \\
m
\end{array}\right)(-1)^{m+1}\left(\left(\frac{\alpha}{\beta}\right)^{m}+1\right)\right]\left(\frac{W_{\alpha, \beta}^{k, \delta} f(z)}{z}\right)^{\gamma}} \\
& +\sigma \sum_{m=1}^{k}\left(\begin{array}{c}
k \\
m
\end{array}\right)(-1)^{m+1}\left(\left(\frac{\alpha}{\beta}\right)^{m}+1\right)\left(\frac{W_{\alpha, \beta}^{k, \delta} f(z)}{z}\right)^{\gamma}\left(\frac{W_{\alpha, \beta}^{k, \delta+1} f(z)}{W_{\alpha, \beta}^{k, \delta} f(z)}\right)
\end{aligned}
$$

be univalent in $\mathbb{U}$. If

$$
\begin{aligned}
& q_{1}(z)+\frac{\sigma}{\gamma} z q_{1}^{\prime}(z) \\
& \prec\left[1-\sigma \sum_{m=1}^{k}\left(\begin{array}{c}
k \\
m
\end{array}\right)(-1)^{m+1}\left(\left(\frac{\alpha}{\beta}\right)^{m}+1\right)\right]\left(\frac{W_{\alpha, \beta}^{k, \delta} f(z)}{z}\right)^{\gamma} \\
& +\sigma \sum_{m=1}^{k}\left(\begin{array}{c}
k \\
m
\end{array}\right)(-1)^{m+1}\left(\left(\frac{\alpha}{\beta}\right)^{m}+1\right)\left(\frac{W_{\alpha, \beta}^{k, \delta} f(z)}{z}\right)^{\gamma}\left(\frac{W_{\alpha, \beta}^{k, \delta+1} f(z)}{W_{\alpha, \beta}^{k, \delta} f(z)}\right) \\
& \prec q_{2}(z)+\frac{\sigma}{\gamma} z q_{2}^{\prime}(z),
\end{aligned}
$$

then

$$
q_{1}(z) \prec\left(\frac{W_{\alpha, \beta}^{k, \delta} f(z)}{z}\right)^{\gamma} \prec q_{2}(z)
$$

and $q_{1}$ and $q_{2}$ are, respectively, the best subordinant and the best dominant.

Theorem 6. Let $q_{1}$ and $q_{2}$ be convex univalent in $\mathbb{U}$ with $q_{1}(0)=q_{2}(0)=1$. Suppose $q_{1}$ satisfies (22) and $q_{2}$ satisfies (13). Let $f \in \mathcal{A}$ satisfies

$$
\left(\frac{W_{\alpha, \beta}^{k, \delta+1} f(z)}{W_{\alpha, \beta}^{k, \delta} f(z)}\right)^{\gamma} \in H[1,1] \cap Q
$$

and $\Omega(\eta, \tau, \gamma, \lambda, k, \delta, \alpha, \beta ; z)$ is univalent in $\mathbb{U}$, where $\Omega(\eta, \tau, \gamma, \lambda, k, \delta, \alpha, \beta ; z)$ is given by (15). If

$$
\begin{aligned}
\eta+\tau q_{1}(z)+\lambda \frac{z q_{1}^{\prime}(z)}{q_{1}(z)} & \prec \Omega(\eta, \tau, \gamma, \lambda, k, \delta, \alpha, \beta ; z) \\
& \prec \eta+\tau q_{2}(z)+\lambda \frac{z q_{2}^{\prime}(z)}{q_{2}(z)},
\end{aligned}
$$


then

$$
q_{1}(z) \prec\left(\frac{W_{\alpha, \beta}^{k, \delta+1} f(z)}{W_{\alpha, \beta}^{k, \delta} f(z)}\right)^{\gamma} \prec q_{2}(z)
$$

and $q_{1}$ and $q_{2}$ are, respectively, the best subordinant and the best dominant.

Remark 2. By selecting the particular values of $\delta, k, \alpha$ and $\beta$, we can derive a number of known results. Some of them are given below:

(1) Taking $\delta=0$ in Theorem 1, we obtain the results obtained by $\mathrm{Mu}-$ rugusundaramoorthy and Magesh [10, Corollary 3.3],

(2) Putting $k=1, \alpha=1-\beta$ and $\beta \geq 0$ in Theorems 1,3 and 5 , we get the results obtaioned by Răducanu and Nechita [11, Theorem 3.1, Theorem 3.6, Theorem 3.9],

(3) Setting $\alpha=0$ and $k=\beta=1$ in Theorems 1, 3 and 5, we get the results obtained by Răducanu and Nechita [11, Corollary 3.3, Corollary 3.8, Corollary 3.11].

\section{REFERENCES}

[1] J. W. Alexander, Functions which map the interior of the unit circle upon simple region, Annals of Mathematics, 17 (1) (1915), 12-22.

[2] F. M. Al-Oboudi, On univalent functions defined by a generalized Sălăgean operator, International Journal of Mathematics and Mathematical Sciences, 27 (2004), 1429-1436.

[3] A. A. Attiya, M. F. Yassen, Some subordination and superordination results associated with generalized Srivastava-Attiya operator, Filomat, 31 (1) (2017), 53-60.

[4] S. D. Bernardi, Convex and starlike univalent functions, Transactions of the American Mathematical Society, 135 (1969), 429-446.

[5] T. Bulboacă, Classes of first order differential superordinations, Demonstratio Mathematica, 35 (2) (2002), 287-292.

[6] N. E. Cho, H. M. Srivastava, Argument estimates of certain analytic functions defined by a class of multiplier transformations, Mathematical and Computer Modelling, 37 (1-2) (2003), 39-49.

[7] I. B. Jung, Y. C. Kim, H. M. Srivastava, The Hardy space of analytic functions associated with certain one-parameter families of integral operators, Journal of Mathematical Analysis and Applications, 176 (1993), 138-147.

[8] S. S. Miller, P. T. Mocanu, Differential Subordinations: Theory and Applications, Series on Monographs and Textbooks in Pure and Applied Mathematics Vol. 225, Marcel Dekker Inc., New York and Basel, 2000.

[9] S. S. Miller, P. T. Mocanu, Subordinants of differential superordinations, Complex Variables, 48 (10) (2003), 815-826.

[10] G. Murugusundaramoorthy, N. Magesh, Differential subordinations and superordinations for analytic functions defined by Dziok-Srivastava linear operator, Journal of Inequalities in Pure and Applied Mathematics, 7 (4) (2006), Article 152, 1-20. 
[11] D. Răducanu, V. O. Nechita, A differential sandwich theorem for analytic functions defined by the generalized Salagean operator, Australian Journal of Mathematical Analysis and Applications, 9 (1) (2012), 1-7.

[12] S. Rahrovi, Subordination and superordination properties for convolution operator, International Journal of Nonlinear Analysis and Applications, 6 (2) (2015), 137-147.

[13] G. St. Sălăgean, Subclasses of univalent functions, Lecture Notes in Mathematics, Springer Verlag, Berlin, 1013 (1983), 362-372.

[14] T. M. Seoudy, Subordination and superordination results of p-valent analytic functions involving a linear operator, Boletim da Sociedade Paranaense de Matematica, 35 (2) (2017), 223-234.

[15] Y. J. Sim, O. S. Kwon, N. E. Cho, H. M. Srivastava, Bounds for the Real Parts and Arguments of Normalized Analytic Functions Defined by the Srivastava-Attiya Operator, Journal of Computational Analysis and Applications, 28 (4) (2020), 628-645.

[16] H. M. Srivastava, A. A. Attiya, An integral operator associated with the HurwitzLerch Zeta function and differential subordination, Integral Transforms and Special Functions, 18 (3) (2007), 207-216.

[17] H. M. Srivastava, S. Gaboury, A new class of analytic functions defined by means of a generalization of the Srivastava-Attiya operator, Journal of Inequalities and Applications, 2015 (2015), ArticleID 39, 1-15.

[18] H. M. Srivastava, S. Gaboury, F. Ghanim, A unified class of analytic functions involving a generalization of the Srivastavâă $\breve{S} A t t i y a$ operator, Applied Mathematics and Computation, 251 (2015), 35-45.

[19] H. M. Srivastava, A. S. Juma, H. M. Zayed, Univalence conditions for an integral operator defined by a generalization of the Srivastava-Attiya operator, Filomat, 32 (6) (2018), 2101-2114.

[20] H. M. Srivastava, A. Prajapati, P. Gochhayat, Third order differential subordination and differential superordination results for analytic functions involving the SrivastavaAttiya operator, Applied Mathematics and Information Sciences, 12 (3) (2018), 469-481.

[21] H. M. Srivastava, A. K. Wanas, Strong differential sandwich results of $\lambda$-PseudoStarlike Functions with Respect to Symmetrical Points, Mathematica Moravica, 23 (2) (2019), 45-58.

[22] S. R. Swamy, Inclusion properties of certain subclasses of analytic functions, International Mathematical Forum, 7 (36) (2012), 1751-1760.

[23] B. A. Uralegaddi, C. Somanatha, Certain classes of univalent functions, in Current Topics in Analytic Function Theory, (Edited by H. M. Srivastava and S. Own), 371-374, World Scientific, Singapore, 1992.

[24] A. K. Wanas, New differential operator for holomorphic functions, Earthline Journal of Mathematical Sciences, 2 (2) (2019), 527-537. 
[25] A. K. Wanas, A. H. Majeed, Differential sandwich theorems for multivalent analytic functions defined by convolution structure with generalized hypergeometric function, Analele Universitatii din Oradea. Fascicola matematica, XXV (2) (2018), 37-52.

\author{
Abbas Kareem Wanas \\ Department of Mathematics \\ College of Science \\ UNIVERSITY OF AL-QADISIYAH \\ IRAQ \\ E-mail address: abbas.kareem.w@qu.edu.iq
}

Gangadharan Murugusundaramoorthy

Department of Mathematics

School of Advanced Sciences

Vellore Institute of Technology

DeEmed to Be University

VELLORE-632014

INDIA

E-mail address: gmsmoorthy@yahoo.com 